\title{
Campañas políticas y desafección ciudadana: aproximación desde Chile a los efectos de las actividades electorales en el proceso democrático
}

\section{Alberto Pedro López-Hermida Russo ${ }^{1}$ Pedro Fierro-Zamora ${ }^{2}$}

Recibido: 2015-06-26

Enviado a pares: 2015-07-14
Aprobado por pares: 2015-09-02

Aceptado: 2015-09-22

DOI: 10.5294/pacla.2016.19.2.2

Para citar este artículo / To reference this article / Para citar este artigo López-Hermida Russo, A. P. \& Fierro-Zamora, P. (Junio de 2016). Campañas políticas y desafección ciudadana: aproximación desde Chile a los efectos de las actividades electorales en el proceso democrático. Palabra Clave, 19(2), 365-397. DOI: 10.5294/pacla.2016.19.2.2

\section{Resumen}

La creciente desafección política — demostrada en urnas y estudios demoscópicos-parece ser un fenómeno compartido por distintos países, donde Chile no ha quedado al margen. En este contexto, ¿pueden analizarse las campañas electorales como herramientas que aporten en la solución de esta problemática? El abordaje de esta pregunta exige un modelo distinto a la hora de pensar los procesos electorales, con la consecuente irrupción de nuevos interrogantes que vayan más allá de la simple participación electoral. En este sentido, gran parte de la literatura ha tratado sobre la incidencia real de las campañas en la decisión de votar y en la dirección de esa preferencia. Sin embargo, el interés no ha sido el mismo respecto de los efectos de las campañas en el proceso democrático en su sentido más amplio y el escenario actual exige fortalecer esta área. En la presente investigación, se

Universidad de los Andes, Chile. alhr@uandes.cl

Universidad Adolfo Ibáñez, Escuela de Negocios, Chile.pedro.fierro@uai.cl 
busca precisamente un énfasis en esto último, tratando de identificar y rescatar el papel que cumplen las campañas en el fortalecimiento de la democracia y en la disminución de la desafección política. Para reconstruir una revisión histórica sobre las campañas, llegaremos al concepto de 'eficacia política', el cual utilizaremos como base conceptual para analizar con solidez teórica el estado actual de Chile. Finalmente, se destacarán los efectos que las campañas electorales pueden tener en el grado de eficacia política, lo cual nos permitirá conocer - con evidencia comparada concreta- el eventual papel que tendría esta actividad en el fortalecimiento de la democracia chilena.

\section{Palabras clave}

Comunicación política; elecciones; democracia; desafección (Fuente: Tesauro de la Unesco). 


\section{Political Campaigns and Public Disaffection: An Approach from Chile to the Effects of Electoral Activities in the Democratic Process}

\section{Abstract}

The growing political disaffection - shown in polls and opinion polls- appears to be shared by different countries, where Chile has not been left out of phenomenon. In this context can they analyze the electoral campaigns as tools that contribute in solving this problem? Addressing this question requires a different model of thinking when electoral processes, with the consequent emergence of new questions that go beyond mere electoral participation. In this sense, much of the literature has discussed the real impact of campaigns in the decision to vote and in the direction of that preference. However, interest has not been the same with respect to the effects of the campaigns in the democratic process in its broadest sense and the current scenario requires strengthening the edge. In this research, it precisely seeks an emphasis on the latter, trying to identify and rescue the role campaigns in strengthening democracy and reduce political disaffection. To reconstruct a historical review of the campaigns we reach the concept of 'political efficacy', which we will use as a conceptual basis for theoretical soundness to analyze the current state of Chile. Finally, the effects that the campaign may have on the level of political efficacy will be highlighted, allowing us to know, with comparative evidence specifically the potential role that this activity would have in strengthening Chilean democracy.

\section{Keywords}

Political communication; elections; democracy; disaffection (Source: Unesco Thesaurus). 


\section{Campanhas políticas e descontentamento cidadão: aproximação do Chile aos efeitos das atividades eleitorais no processo democrático}

\section{Resumo}

O crescente descontentamento político — demonstrado nas urnas e estudos demoscópicos — parece ser um fenômeno compartilhado por diferentes países, onde o Chile não ficou atrás. Nesse contexto, podem ser analisadas as campanhas eleitorais como ferramentas que contribuem para a solução dessa problemática? A abordagem dessa pergunta exige um modelo diferente no momento de pensar os processos eleitorais, com a consequente irrupção de novos interrogantes que vão mais além da simples participação eleitoral. Nesse sentido, grande parte da literatura vem tratando sobre a incidência real das campanhas na decisão de votar e na direção dessa preferência. Contudo, o interesse não tem sido o mesmo a respeito dos efeitos das campanhas no processo democrático em seu sentido mais amplo, e o cenário atual exige fortalecer essa aresta. Nesta pesquisa, busca-se justamente uma ênfase nisto ao tentar identificar e resgatar o papel que as campanhas cumprem no fortalecimento da democracia e na diminuição do descontentamento político. Para reconstruir uma revisão histórica sobre as campanhas, chegaremos ao conceito de "eficácia política", o qual utilizaremos como base conceitual para analisar com solidez teórica o estado atual do Chile. Finalmente, serão destacados os efeitos que as campanhas eleitorais podem ter no grau de eficácia política, que nos permitirá conhecer — com evidência comparada concreta - o eventual papel que teria essa atividade no fortalecimento da democracia chilena.

\section{Palavras-chave}

Comunicação política; eleições; democracia; descontentamento (Fonte: Tesauro da Unesco). 


\section{Campañas políticas, crisis y desafección}

El escenario político actual en prácticamente todos los países occidentales exige una mirada sistémica a la hora de evaluar la actividad política y el papel de la sociedad en las problemáticas que nos aquejan en relación al proceso de toma de decisiones. En Chile, por ejemplo, diversas autoridades -incluso a la Presidenta de la República - han reconocido la existencia de una "crisis de confianza", producto de diversos escándalos de corrupción, que han sido evidenciados en el último tiempo. Estos sucesos no son extraños en la actualidad iberoamericana, pero sí novedosos en la tradición política de este país.

En un contexto de crisis, la desafección política siempre es un objeto interesante de estudio a la hora de realizar un análisis adecuado del funcionamiento de las instituciones y de los procesos políticos.

Sin embargo, es importante ser justos al reconocer la existencia de índices que nos demuestran que los grados de confianza en países occidentales, como Chile, se encontraban en declive, incluso desde antes de la "crisis" actual. Pese a esto, parece indudable que los conflictos recientes le han otorgado mayor visibilidad pública al problema. En este sentido, por ejemplo, son elocuentes los resultados obtenidos durante los últimos años en el estudio demoscópico Latinobarómetro, los cuales permiten realizar un análisis comparado de la situación chilena respecto del resto del continente. Con estos datos, por ejemplo, no resulta muy aventurado sostener que la desconfianza en los procesos de toma de decisión en Chile parece estar más deteriorada que en otros países del vecindario, como tampoco afirmar que las cifras se vienen manteniendo desde hace varios años.

La situación particular del último tiempo, así como los hechos de corrupción que han desencadenado esta nueva "crisis de confianza", han llevado a ciertos agentes a poner en duda la legitimidad de instituciones o procesos, y cuando esto sucede resulta sensato que los interesados en comunicación nos cuestionemos el papel que debiesen cumplir las campañas políticas en democracia. Así es como han existido diversos esfuerzos por tratar de definir y determinar los fines y las funciones de una campaña 
electoral y, en definitiva, medir su relevancia para la conformación de una ciudadanía participativa e informada (Freedman, Franz y Goldstein, 2004; Hansen y Pedersen, 2014). En definitiva, este trabajo indagará en una alternativa distinta respecto del papel que podrían cumplir las campañas en un contexto de crisis y desafección.

\section{Evolución en el estudio de los efectos de las campañas políticas}

Para entender la evolución del estudio de los efectos y las funciones de las campañas electorales, debemos necesariamente remontarnos a las investigaciones de principio del siglo pasado, muy marcadas por el nacimiento de la propaganda, como hoy la entendemos. Si bien es cierto que la mayoría de estas investigaciones tenían como objeto de estudio la opinión pública y la comunicación de masas, encontraremos conclusiones muy relevantes para el entendimiento de la comunicación política en general, sobre todo si consideramos que hoy en día gran parte de las campañas descansan esencialmente en herramientas masivas (García, 2004). En este contexto, surge el trabajo de Lippmann (1922), quien en la década de 1920 revoluciona el concepto de 'opinión pública'. Para el autor, siempre se ha asumido que el sistema requiere ciudadanos de buen corazón, mente razonable y juicio equilibrado, pero sostiene a la vez que nos ha faltado preguntarnos la forma en que lograremos nutrir la mente y el corazón que requiere un buen ciudadano. En este sentido, Lippmann señala que, "para funcionar de manera competente y honesta, el sistema habría tenido que asumir que todos los hombres tendrían que conformarse con obtener una experiencia muy parcial de los asuntos públicos" (p. 216). De esta forma, queda en evidencia su clara visión respecto de la insuficiente información objetiva que recibían los ciudadanos para tomar decisiones sobre asuntos públicos de la manera en que lo requería la teoría democrática de la opinión pública.

Algunos años después, durante el periodo de postguerra, cobra relevancia el trabajo de Joseph Klapper, quien, en cierta medida, venía a confirmar la poca incidencia que la comunicación de masas, en la cual muchas veces enmarcamos la mayor parte de las herramientas electorales, tenía respecto al cambio de opiniones previas (Klapper, 1960; Rodríguez-Po- 
lo, 2011). En este sentido, se vino a sostener que la mayor parte de los esfuerzos de comunicación tenían como resultado un efecto de refuerzo de lo que ya se pensaba, determinado por una serie de factores, tales como las predisposiciones, la naturaleza de los medios, los grupos y sus normas, entre otros (Klapper, 1960; Moreno, 2010). Ya con el trabajo que realizaron Paul Lazarsfeld, Bernard Berelson y Hazel Gaudet en The People's Choice, de 1944, se hablaba del "efecto mínimo" de las campañas políticas, lo que Klapper vino a reforzar con su investigación.

Solo luego de esto - y asumiendo las limitaciones propias de la comunicación de masas tratadas por Lazarsfeld, Berelson y Gaudet (1948), Klapper (1960) y Lippmann (1922) — comienzan a aparecer objetos de estudios que fueron precisamente respaldando la posibilidad de efectivamente persuadir al votante (García, 2004).

De forma paralela, se comienza a hablar también de una mirada sistémico-institucional de las campañas políticas, identificando que el mismo sistema electoral y la cultura de cada país incidirían directamente en la gestión de las actividades electorales, comenzando a ver al votante ya no solo como individuo, sino también en su contexto social (Canel, 1998; Maarek, 2011).

\section{Resabios conceptuales en el análisis actual de las campañas electorales: persuadir, informar y movilizar}

Independientemente de la discusión y evolución académica que ha existido en el estudio de las campañas electorales y sus efectos o funciones, es esperable que busquemos encontrar parámetros más o menos estandarizados para comenzar un debate productivo respecto de ellas. En esta lógica, si quisiéramos conceptualizar lo que entendemos por campaña, sería sensato partir con el mismo Lippmann (1922), quien las concibe como la actividad donde "se practica el arte de inducir a todo tipo de personas que piensan diferente a votar lo mismo" (p. 172). Para lograr este objetivo, el autor plantea la necesidad de identificar grupos de interés y emplear un discurso para todos ellos. Algunos años después, Lazarsfeld, Berelson y Gaudet (1948) 
las definen como "el proceso de planificar y ejecutar actividades con la intención de ganar votos" (citado en García, 2004, p. 7). Y más adelante, el profesor Christhopher Arterton (1987) las ha definido como "esfuerzos encaminados a dirigir mensajes persuasivos a un vasto electorado" (citado en en García, 2004, p. 7).

Como podemos observar, contamos con, al menos, tres definiciones que en más de sesenta años comparten elementos esenciales y que se han expandido hasta investigaciones recientes. Siguiendo la misma línea de estas conceptualizaciones, para un grupo de académicos la investigación sobre los eventuales efectos de las campañas electorales se han centrado en básicamente tres puntos esenciales: persuadir, informar y comprometer $(\mathrm{Hu}-$ ber y Arceneaux, 2007; Freedman, Franz y Goldstein, 2004; Goldstein y Ridout, 2004), lo cual precisamente queda en evidencia con la forma en que Pippa Norris define las campañas varios años más tarde —en2002—, sosteniendo que se tratan de "esfuerzos organizados para informar, persuadir y movilizar" (2002, p. 120).

Todo tiene bastante lógica si consideramos que por mucho tiempo se entendieron los efectos de las campañas electorales como una distinción entre cogniciones, afectos y acciones (Canel, 1998), conceptos que lograron permanecer como objetos de estudio, pese a la mirada sistémico-institucional que se le pretendía dar a estas actividades políticas.

De esta forma, se han llevado a cabo distintos experimentos para medir el grado de información (cognoscitivo), persuasión (afectivo) o compromiso (acción) que las distintas herramientas de campaña generan en el votante, para determinar si estas importan o no. Los resultados pueden ser dispares y, en cierta medida, la discusión metodológica de los experimentos ejecutados puede ser tan interesante como la teórica, pero, sin embargo, las conclusiones obtenidas de estos trabajos han elevado en gran medida la investigación respecto de ciertos puntos de las campañas.

En este sentido, algunos autores han demostrado que las campañas políticas no necesariamente logran comprometer a los ciudadanos con una candidatura en específico, sobre todo si consideramos la publicidad electo- 
ral aisladamente (Huber y Arceneaux, 2007). Pese a esto, otros académicos defienden mediante distintas metodologías la incidencia positiva que tienen las herramientas publicitarias en la participación electoral (Freedman, Franz y Goldstein, 2004), argumentando que la preocupación por elecciones venideras aumenta en consideración al grado de exposición que los distintos grupos tengan.

Respecto del carácter informativo que las herramientas de publicidad electoral generan en el electorado, las opiniones también son diversas. Mientras algunos académicos sostienen que las campañas tienden a disminuir las brechas de conocimientos en el periodo electoral entre los grupos con mayor o menor conocimiento político (Hansen y Pedersen, 2014; Freedman, Franz y Goldstein, 2004), otros revelan la falta de evidencia para sacar conclusiones claras al respecto (Huber y Arceneaux, 2007).

\section{Persuasión}

Una gran cantidad de autores han sido enfáticos al sostener que las campañas importan (Goldstein y Ridout, 2004; Freedman, Franz y Goldstein, 2004; Huber y Arceneaux, 2007; Hansen y Pedersen, 2014), pero aun así reconocen las dificultades para distinguir en términos concretos qué es lo que persuade, informa o moviliza (Goldstein y Ridout, 2004).

En el mismo camino de determinar el grado de incidencia de las campañas, existe evidencia de que, por ejemplo, los anuncios de televisión afectan en lo cognoscitivo, afectivo y en el comportamiento del votante, pero también de que, salvo en el votante indeciso, no cambia la decisión de voto, sino que más bien reafirma posturas (Canel, 1998).

Siguiendo en esta misma línea, según Moreno (2010), es indispensable para el entendimiento de las campañas políticas distinguir al menos tres funciones propias de estas actividades: la persuasión, la legitimación y la selección de las élites políticas. Bajo esta perspectiva, la única función que buscarían directamente los partidos políticos sería la primera, siendo ahí donde radican los efectos de activación, refuerzo y con- 
versión, ampliamente estudiados desde Lazarsfeld, Berelson y Gaudet (1948) en adelante. ${ }^{3}$

Precisamente, esta puede ser una de las razones por la cual gran parte de la investigación sobre los efectos de las campañas se haya volcado al grado de incidencia de estas acciones en la dirección del voto, aunque quizá sin resultados concluyentes. Si analizamos, por ejemplo, las definiciones de campañas políticas tratadas, podremos apreciar que el carácter persuasivo es elemento esencial de cada una, lo cual tendría cierta lógica si estudiáramos esta actividad política desde la maximización de votos, entendiendo que el sentido último - y quizá único- sea ganar una determinada elección. Pero claramente este no es nuestro caso, pues creemos que el papel que pueden desempeñar en la democracia las campañas abarcan algo más que la acumulación de preferencias.

En este mismo sentido, Goldstein y Ridout (2004) son claros al señalar que los grandes avances respecto de la investigación se han dado mayormente en lo relacionado con lo que ellos llaman los "efectos indirectos" de las campañas electorales, es decir, en cuanto a la información generada y al impacto en urnas. Esta situación no ha sido de la misma forma con la incidencia en el objetivo de los patrocinadores: incidir en la dirección del voto y ganar elecciones.

Como hemos mencionado, Moreno (2010) habla de dos funciones distintas de la de la persuasión y que son una consecuencia no querida directamente por los partidos, nos referimos a la legitimación y a la selección de élites políticas, lo cual se relaciona, en cierto sentido, con la distinción que seguimos en este trabajo, pues precisamente la función de legitimación incluiría los efectos relacionados con el grado de información traspasada a la ciudadanía y la movilización efectiva respecto de la participación electoral, aunque nos interesa ir algo más allá.

3 Martínez i Coma (2005) plantea ampliar estos tres efectos típicos estudiados por Lazarsfeld, Berelson y Gaudet (1948) y agregar la desactivación, es decir, votantes que perdieron su existente intención de votar debido a un efecto desmovilizador de las campañas políticas, lo cual se relacionaría con el efecto de las campañas en la participación electoral más que en la persuasión, de lo que trataremos más adelante al hablar de la generación de compromiso. 
Pese a lo planteado por Goldstein y Ridout (2004) — sumando la distinción entre funciones de Moreno (2010) basada esencialmente en el trabajo realizado por Lazarsfeld, Berelson y Gaudet (1948) en la década de 1940_, la amplia literatura respecto de los efectos de esta actividad no parece realizar una distinción previa entre funciones queridas o no queridas por los partidos políticos, sino que, como hemos mencionado, más bien analizan genéricamente el compromiso, la información y la persuasión, como efectos propios de la actividad electoral.

\section{Información}

La gran pregunta desde el punto de vista de Lippmann (1922) sería cómo nutriremos finalmente la mente y el corazón para contar con una ciudadanía digna de participar en las decisiones de los asuntos públicos. A este interrogante se suma luego Lazarsfeld, Berelson y Gaudet (1948), quien también reconocía que solo algunos ciudadanos logran alcanzar el grado de conocimiento necesario.

Es aquí donde el trabajo de Freedman, Franz y Goldstein (2004) toma relevancia, pues, de alguna forma, vuelven a plantearse dudas similares a las realizadas por Lippmann (1922) y Lazarsfeld, Berelson y Gaudet (1948) respecto de cómo ciudadanos con poco interés y conocimiento sobre política pueden ejecutar un juicio más o menos razonable. Precisamente, los autores concluyen, luego de la ejecución de una interesante metodología de levantamiento de información, que los treinta segundos estadounidenses de publicidad televisiva en época electoral se han transformado en una de las mayores fuentes de información para la ciudadanía (Freedman, Franz y Goldstein, 2004), haciéndola en definitiva más atenta, informada y participativa.

Lo concluido por Freedman, Franz y Goldstein (2004) se condice con otros autores que rescatan el valor informativo que cumplen las campañas electorales y, en específico, las herramientas de publicidad política mayormente utilizadas (Arceneaux, 2006; Atkin y Held, 1976; Hansen y Pedersen, 2014; Brians y Wattenberg, 1996; Coleman y Manna, 2000). 
En este mismo sentido, Brians y Wattenberg (1996) también defienden el potencial informativo que tiene la publicidad política, incluso la negativa. De esta forma, y pese a aquellos estudios que sostienen que la publicidad negativa podría tener un efecto reprochable en términos emotivos respecto de la ciudadanía (Ansolabehere, Iyengar y Simon, 1999), los autores plantean que aportan en la obtención de información precisa y como una herramienta activa en la evaluación de candidatos y temáticas, conclusiones que se verían respaldadas, de alguna forma, por autores, como Kahn y Kenney (2000).

Los resultados de estas investigaciones son, sin duda, significativos, pero si seguimos los planteamientos de Lippmann (1922) y Lazarsfeld, Berelson y Gaudet (1948), es sensato que aun así nos cuestionemos el nivel de información que suelen tener aquellos que participan del proceso electoral. En este punto, cobran importancia investigaciones, como la de Lau y Redlawsk (1997), quienes se hacen cargo precisamente de cuánta información sería necesaria para poder hablar de un voto consciente por parte de los ciudadanos. Los autores parten de la base de que los ciudadanos cumplirían sus deberes democráticos si, al menos, votaran correctamente la mayoría de las veces. Es precisamente esto lo que comprueban con su investigación, concluyendo que mayoritariamente tendemos a votar de la misma forma de la que lo haríamos si contáramos con información plena.

Respecto de la importancia de la información en la definición de la dirección del voto, la investigación de Lau y Redlawsk (1997) puede ser de mucha importancia, pues nos alejan de la teoría clásica respecto del conocimiento requerido para hacer razonablemente buenas decisiones. Este diagnóstico es también compartido por Key (1966), Florina (1981) y Popkin (1991), quienes también señalan que la información suele ser suficiente para tomar una decisión correcta.

\section{Participación electoral}

En algunos estudios sobre los efectos de las campañas en la participación electoral, se suele hablar de generación de compromiso o engagement, lo cual estaría directamente relacionado con lo que Canel (2010) denomi- 
na acción y con lo que Lazarsfeld, Berelson y Gaudet (1948) identifica como activación.

Lo anterior representa una primera dificultad, pues, al hablar de engagement o compromiso estamos suponiendo que el efecto que analizamos tiene incidencia tanto en la decisión de votar como en la dirección de esa preferencia. Pese a que no siempre se suele realizar una distinción entre estas dos consecuencias, lo que nos interesará más bien en este trabajo dice relación con la mera intencionalidad de votar, independientemente de si la activación es favorable o contraria al agente que realiza la campaña. Solo por este motivo preferimos hablar de participación en términos genéricos y no de engagement o compromiso.

Precisamente, el efecto de las campañas en la participación electoral es uno de los que más discusión genera tanto en lo metodológico como en el resultado de las investigaciones.

Freedman, Franz y Goldstein (2004) parecen claros al sostener que las campañas tienen un efecto positivo en la asistencia a urnas, principalmente bajo el argumento de que este tipo de actividad política incrementa el interés del votante en la elección y el sentido de sentirse involucrado, sobre todo por el carácter informativo de las distintas herramientas que suelen utilizarse, lo cual incluso es expandido por Brians y Wattenberg (1999) a las campañas negativas.

Sin embargo, Ansolabehere, Iyengar y Simon (1999) plantean justamente lo contrario, afirmando que el efecto de las campañas negativas en periodo electoral suele ser contraproducente para quienes las emplean, provocando una desactivación y disminuyendo la intención de votar.

Finalmente, autores como Ashworth y Clinton (2007) presentan una posición más bien escéptica, negando el efecto que las campañas puedan tener en la decisión real de votar. Algo parecido a lo que plantean Krasno y Green (2008) ante la inexistencia, según los autores, de indicios claros que avalen la incidencia de publicidad negativa o positiva en participación. 


\section{Replanteamiento de los efectos de las campañas electorales en un contexto democrático: algo más que el voto}

En un contexto de crisis de confianza y desafección política, ¿podemos reducir la función y los efectos de las campañas al persuadir, informar y gatillar la participación electoral?

Ya famosa es la opinión de McGinnis (1968) respecto de que Nixon fue vendido como si se tratara de un producto de consumo, lo cual precisamente evidencia la exclusividad que, en muchos análisis, otorgamos a esta función o efecto principal de las campañas y la comunicación política en general: maximizar votos y ganar elecciones.

Pese a esta mirada ofrecida, no podemos negar que, en la actualidad, ha existido un interés por estudiar los efectos de la actividad electoral en el proceso democrático.

Así es precisamente como Moreno (2010) plantea una función de legitimidad de las campañas, asegurando que "tiene implicaciones diferentes según se considere desde la perspectiva de los partidos políticos, o desde el punto de vista de la teoría de la democracia” (p.55). De esta misma forma, Goldstein y Ridout (2004) mencionan los efectos indirectos de la actividad electoral. Algo parecido sucede con el estudio ya mencionado de Freedman, Franz y Goldstein (2004), quienes sostienen que la información generada por las acciones de campaña fomentan una sociedad atenta, informada y participativa. Sin embargo, en todos estos estudios, se suele profundizar el análisis de la información generada o el nivel de incidencia en la participación electoral, y en muy pocos casos se ha ampliado a una mirada integral o sistémica respecto del aporte sustantivo a la legitimidad de los procesos democráticos.

En este sentido, quizá un caso distinto podría ser Pippa Norris (2001, 2002), aunque debemos reconocer que su investigación ha tenido un enfoque bastante distinto, pues se ha centrado más bien en la inexistencia de 
relación causal entre herramientas de campañas modernas y el aumento de los índices de cinismo, desafección e ignorancia. Pese a eso, la autora ha llegado a conclusiones interesantes respecto de la relación positiva de las campañas en cuanto a los niveles de compromiso cívico. Aun así, la profesora Norris opta por reconducir el civic engagement a indicadores relacionados con los efectos históricamente estudiados de las campañas, como la información y participación (Norris, 2001, 2002), aunque esta vez incluyendo la credibilidad como elemento por considerar, lo cual nos ayuda bastante en nuestro propósito.

De esta forma, y en virtud de llegar a dimensiones anteriores a los efectos usualmente estudiados, suscribimos la necesidad de replantearnos los efectos de las campañas políticas en el proceso democrático desde una mirada sistémica y cultural (Canel, 1998; Hansen y Pedersen, 2014).

En este contexto, es fundamental reconocer que el voto no abarca todos los comportamientos políticos (Canel, 1998). La participación ciudadana en los procesos de toma de decisión incluyen diversas acciones y sensaciones que hasta el momento no han sido del todo consideradas en los efectos de las actividades políticas, y específicamente en el contexto de campañas electorales. Esto último nos obliga a considerar estas acciones de forma integral, y no tan solo midiendo sus efectos de acuerdo con el grado de conocimiento generado o con el ganador de un proceso determinado.

Así es como sostenemos que los efectos en el proceso democrático de las campañas electorales son mucho mayores, pero ¿qué dimensiones nos pueden dar luz a la hora de evaluar estos efectos sistémicos en un contexto de apatía política?

\section{Eficacia política interna y externa}

Podemos encontrar distintas alternativas en la búsqueda de una dimensión que nos ayude a generar una mirada sistémica e institucional sobre los efectos de las acciones de campaña. En este sentido, una opción podría haber sido considerar el conocimiento, interés y entendimiento de la política que las actividades electorales despierten en el ciudadano (Norris, 2002), o qui- 
zá la incidencia que tienen en los niveles de credibilidad y satisfacción con la democracia (Banducci y Karp, 2003). Sin embargo, buscábamos acercarnos a índices algo más concretos respecto de la confianza y la cercanía institucional. Así, puede ser interesante dar lectura a Campbell, Gurin y Miller (1954), quienes trabajaron parámetros importantes relacionados con el proceso de decisión de un votante.

En este contexto, los autores entienden que los factores que eventualmente podrían incidir en la conformación del voto son seis:

- Identificación con algún partido político.

- Preocupación por temas de políticas nacionales de gobierno.

- $\quad$ Atracción personal por algún candidato.

- $\quad$ Conformidad con los estándares del grupo de cada uno.

- Sensación de eficacia personal en el área de la política.

- Sensación de obligación cívica de votar.

Independientemente de la pertinencia de esta clasificación, y del amplio debate que ha existido respecto de algunas de estas consideraciones, resulta sensato dividir esta lista enunciada en dos grupos, como de hecho los mismos autores lo hacen. Por un lado, aquellos factores que están relacionados directamente con la decisión de votar y con la dirección de ese voto, los cuales serían los cuatro primeros. Y en segundo lugar, aquellos relacionados directamente solo con la decisión de votar, nos referimos a los dos últimos.

Es precisamente en uno de estos factores finales donde pretendemos poner nuestra atención, específicamente en el concepto de 'eficacia política'.

En la obra de 1957, The voter decides, los autores entienden el concepto de 'eficacia política' como "la sensación de que las acciones políticas individuales tienen, o pueden tener, un impacto en el proceso político", en definitiva, "la sensación de que el cambio político o social es posible, y que el ciudadano puede jugar un rol importante en él” (p. 187). 
Como podemos ver, la conceptualización trabajada por estos autores nos acerca bastante a la determinación del grado de afección política existente y, más aún, al nivel de confianza en el sistema. De esta forma, la eficacia política sería un elemento neutro que instaría a votar, pero que es anterior y superior a la mera participación electoral, lo cual es lo que precisamente nos interesa. Así, si entendiéramos que las campañas inciden en esta variable, implicaría un reconocimiento de que la actividad va más allá de la maximización de preferencias.

\section{Eficacia personal en el área de la política: Chile}

Antes de tratar la eventual incidencia que las campañas podrían tener en los grados de eficacia, y con esto en la desafección ciudadana, nos interesa tener una noción al menos general de la problemática en Chile. En este sentido, el aporte realizado por Campbell, Gurin y Miller (1954) facilita la búsqueda de dimensiones que nos permitan evaluar de mejor manera el contexto chileno.

Para estos efectos, utilizaremos la habitual distinción entre eficacia externa e interna trabajada por distintos autores (Lane, 1959; Converse, 1972; Balch 1974; Acock, Clarke y Stewart, 1985; Finkel, 1985; Niemi, Craig y Mattei, 1991; Morrell, 2003; Lee, 2006; Caprara et al., 2009; Jung et al., 2011; Hansen y Pedersen, 2014), entendiendo como eficacia interna la sensación de que como ciudadanos tenemos las habilidades necesarias para participar en las discusiones políticas, y como eficacia externa la sensación de que el sistema permite que nuestras acciones puedan cambiar en algo la situación actual. Trabajando estos dos conceptos en el escenario nacional, creemos que alcanzaríamos una mirada interesante respecto de los grados de confianza en el sistema e instituciones en el proceso de toma de decisiones.

De esta forma, hemos identificado algunas preguntas de estudios demoscópicos recientes que, a nuestro juicio, tienen directa relación con las definiciones trabajadas en el contexto chileno.

En particular, trabajamos con dos encuestas aplicadas al territorio nacional, el Estudio Latinobarómetro 2011 y la Auditoría a la Democracia 
2012, encuesta elaborada por el Programa de las Naciones Unidas para el Desarrollo (PNUD). De esta forma, y tal como evidencian las tablas 1 y 2 , dimos paso a una clasificación de índices relacionados con eficacia interna y otros con eficacia externa.

Tabla 1. Preguntas respecto de índices de eficacia interna

\begin{tabular}{|c|c|}
\hline Encuesta & Pregunta \\
\hline Auditoría a la Democracia & $\begin{array}{l}\text { 1) Qué tanto está de acuerdo o en desacuerdo con las } \\
\text { siguientes afirmaciones: } \\
\text { a) Siento que tengo una buena comprensión de los asuntos } \\
\text { políticos importantes de Chile. } \\
\text { b) La gente como yo no tiene nada que decir sobre lo que } \\
\text { el gobierno hace. }\end{array}$ \\
\hline Latinobarómetro & $\begin{array}{l}\text { 2) ¿Cuál frase está más cerca de su forma de pensar? } \\
\text { a) La política es tan complicada que no se entiende. } \\
\text { b) La política no es tan complicada y se entiende. }\end{array}$ \\
\hline
\end{tabular}

Tabla 2. Preguntas respecto de índices de eficacia externa

\begin{tabular}{|c|c|}
\hline Encuesta & Pregunta \\
\hline \multirow{6}{*}{$\begin{array}{c}\text { Auditoría a la } \\
\text { Democracia }\end{array}$} & $\begin{array}{l}\text { 1) Existen diferentes opiniones sobre los derechos de las personas en una democracia. En una } \\
\text { escala de } 1 \text { a } 7 \text {, donde } 1 \text { significa que no es importante y } 7 \text { significa que es muy importante, ¿cuán } \\
\text { importante es para usted...? }\end{array}$ \\
\hline & $\begin{array}{l}\text { 2) Vamos a hablar ahora sobre los diputados y senadores del Congreso y a quien ellos representan. } \\
\text { Me gustaría que me dijera ... ¿A quién representan? ¿A quién deberían representar? }\end{array}$ \\
\hline & $\begin{array}{l}\text { 3) Suponga que su municipalidad tomó una decisión que usted considera que es injusta o } \\
\text { perjudicial. En este caso, ¿qué tan probable es que usted, actuando solo o junto con otras personas, } \\
\text { realice algo al respecto? Si hiciera ese esfuerzo, ¿qué tan probable es que su municipalidad le diera } \\
\text { atención a sus demandas? }\end{array}$ \\
\hline & $\begin{array}{l}\text { 4) Si usted tuviera interés en participar y exponer su opinión respecto de proyectos } \\
\text { medioambientales, ¿cree usted que sus demandas serían consideradas por las autoridades? }\end{array}$ \\
\hline & $\begin{array}{l}\text { 5) ¿Qué tanto está de acuerdo o en desacuerdo con las siguientes afirmaciones? } \\
\text { a) ¿No creo que al Gobierno le importe mucho lo que piensa la gente como yo? }\end{array}$ \\
\hline & $\begin{array}{l}\text { 6. ¿Con cuál de las siguientes afirmaciones está usted más de acuerdo? } \\
\text { a) Como uno vota puede influir en el país. } \\
\text { b) Como uno vota no puede influir en lo que pase en el país. }\end{array}$ \\
\hline Latinobarómetro & 7) En términos generales, ¿diría usted que Chile está gobernado para el bien de todo el pueblo? \\
\hline
\end{tabular}

Es importante aclarar que en ningún caso pretendemos obviar las diferencias metodológicas de ambos estudios. Aun así, entendemos que el objetivo de este trabajo nos permite trabajar con diversos indicadores, considerando que el sentido último es simplemente tener una aproximación al 
contexto nacional. De esta forma, y teniendo en cuenta los resultados obtenidos con la aplicación de estas preguntas, llegamos a algunas consideraciones relevantes para nuestro propósito.

En primer lugar, y tal como lo planteaba Campbell, Gurin y Miller (1954) en su trabajo, los datos obtenidos son sugerentes en cuanto a la posibilidad de contar con indicadores claros respecto de los grados de eficacia política, lo cual a la vez permitiría evaluar con cierta certeza los niveles de desafección, entendiéndola siempre como el

\begin{abstract}
sentimiento subjetivo de impotencia, cinismo y falta de confianza en el proceso político, los políticos y las instituciones democráticas que genera un distanciamiento y alienación respecto a éstos, y una falta de interés en la política y los asuntos públicos, pero sin cuestionar el régimen democrático (Torcal, 2003, p. 2).
\end{abstract}

Lo anterior, entre otras cosas, nos permitiría escapar de conceptos válvula para pasar a indicadores medibles desde el punto de vista de la confianza sistémica.

En segundo lugar, los datos también evidencian la necesaria distinción entre eficacia interna y externa antes realizada. Una cosa es la sensación personal de contar con las habilidades necesarias como para participar de las discusiones públicas y otra muy distinta es creer que el sistema permite que mi acción individual cambie el escenario sociopolítico. En este sentido, la distinción sugerida por algunos autores (Lane, 1959; Hansen y Pedersen, 2014) tiene lógica según los resultados obtenidos, lo cual sugiere que las variables que inciden en cada uno también debiesen ser disímiles. Por otro lado, y quizá más interesante, los resultados sugieren ciertas diferencias respecto de la importancia atribuida a las acciones individuales para generar cambios sociopolíticos y a la atribuida al voto como acción individual específica, situación que ya la insinuaba Campbell, Gurin y Miller (1954) en su trabajo. Esta diferencia es concordante con la idea de que la eficacia no se reduce al voto como única acción relevante. Independientemente de que pueda incidir en la activación del votante, ya comentamos la necesidad de entenderla como algo superior y anterior, mucho más cercana a la desafección en su sentido lato. En definitiva, es esto último lo que esencialmente 
nos ocupa en esta oportunidad. Como ya hemos mencionado, bastante se ha dicho sobre la incidencia de las campañas en el aumento de la participación electoral, pero bastante menos respecto de su incidencia en la participación política general.

En tercer lugar, es importante rescatar que los índices que miden eficacia interna y externa variaron en el tiempo (particularmente algunos relacionados con la eficacia externa). Junto con esto, y basados en algunas preguntas rescatadas de la encuesta Latinobarómetro, los resultados también sugieren una diferencia dependiendo del país. En este sentido, el caso de Uruguay es determinante. Precisamente, estas dos últimas consideraciones exigen una mirada más profunda, pues sugieren la existencia de variables que determinan los grados de eficacia existentes, las que pueden ser eventualmente trabajadas (figuras 1 y 2 ).

\section{Figura 1. Encuesta PNUD Auditoría a la democracia}

1. Existen diferentes opiniones sobre los derechos de las personas en una democracia. En una escala de 1 al 7 , donde 1 significa que no es importante y 7 significa que es muy importante, ¿cuán importante es para Ud....?

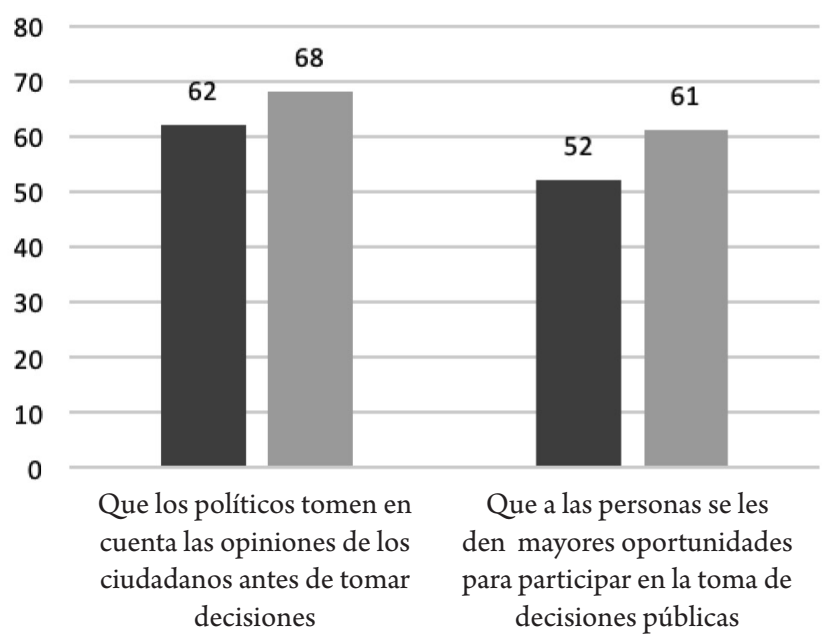

-Nov. 2012 Oct. 2010 
2.1 Vamos a hablar ahora sobre los diputados y senadores del Congreso y a quién ellos representan. Me gustaría que me dijera a quién ellos representan

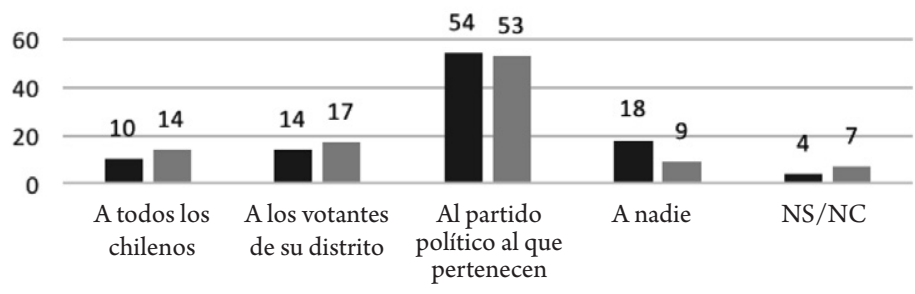

Nov. 2012 Oct. 2010

2.2 Vamos a hablar ahora sobre los diputados y senadores del Congreso y a quién ellos representan. Me gustaría que me dijera a quién ellos deberían representar

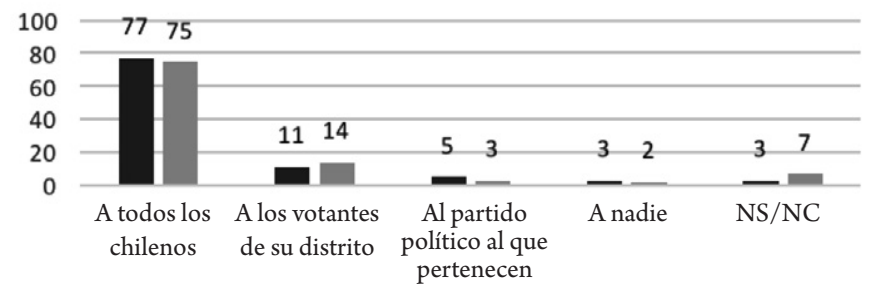

nov. 2012 act. 2010

3. Suponga que su municipalidad tomó una decisión que Ud. considera que es injusta o perjudicial...

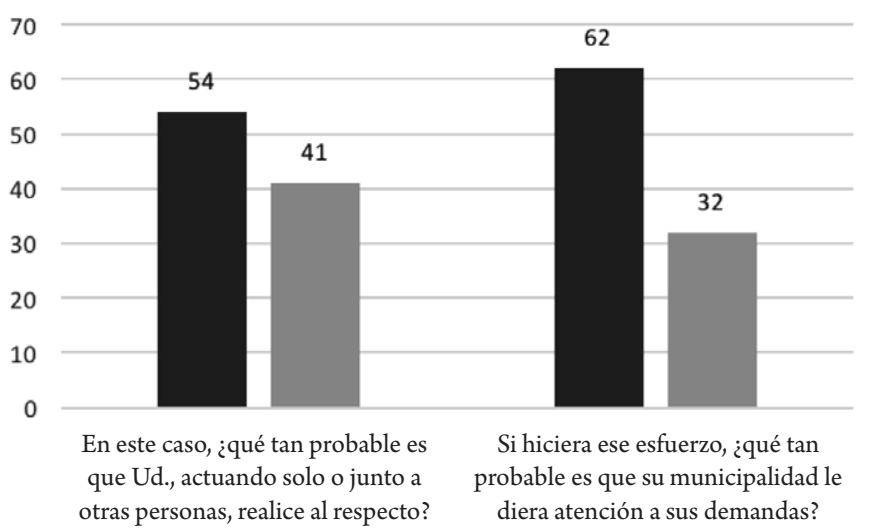

- No es muy probable + No es probable

Muy probable + Probable 
4. Si Ud. tuviera interés en participar y exponer su opinión respecto a proyectos medioambientales

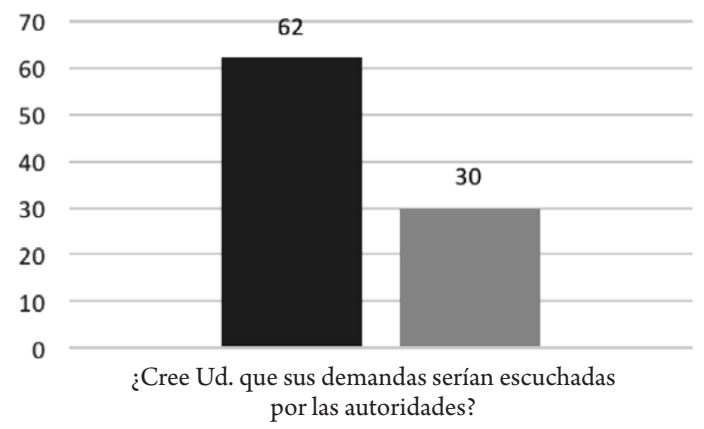

- No es muy probable + No es probable

Es muy probable + Es probable

5. ¿Qué tanto está de acuerdo o en desacuerdo con las siguientes afirmaciones? (\% de acuerdo + muy de acuerdo)

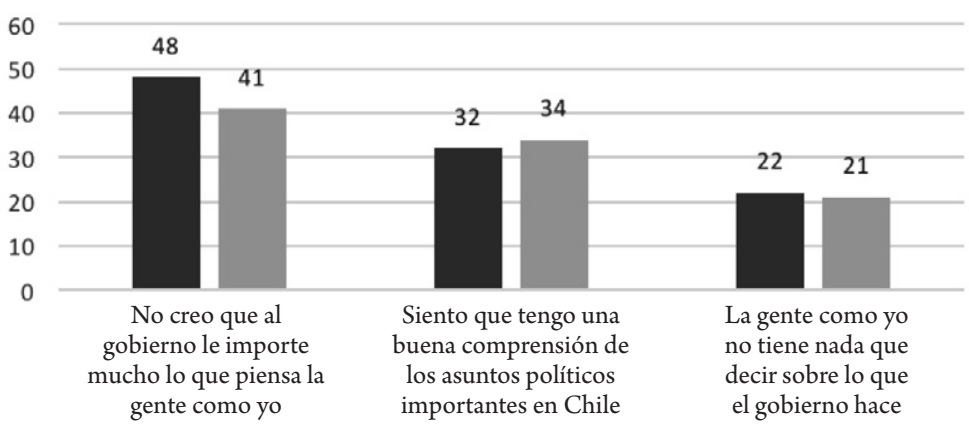

Nov. 2012 Oct. 2010

6. ¿Con cuál de las siguientes afirmaciones está Ud. más de acuerdo?

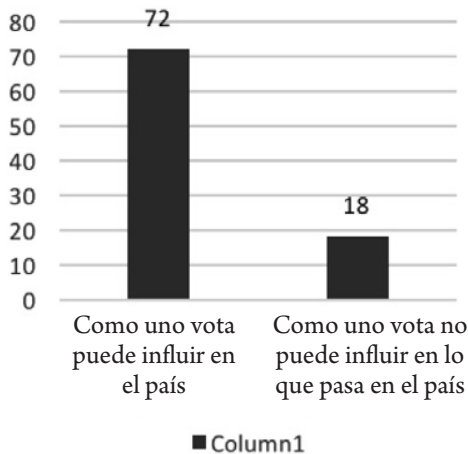




\section{Figura 2. Encuesta Latinobarómetro}

1. En términos generales ¿diría usted que su país está gobernado por unos cuantos grupos poderosos en su propio beneficio, o que está gobernado para el bien

de todo el pueblo?

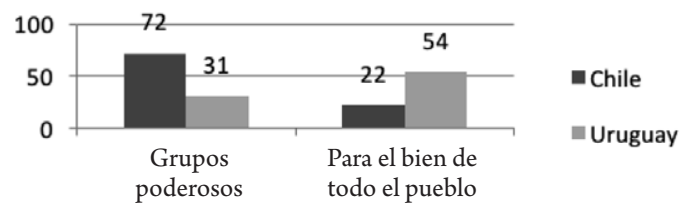

2. ¿Cuál frase está más cerca de su manera de pensar?

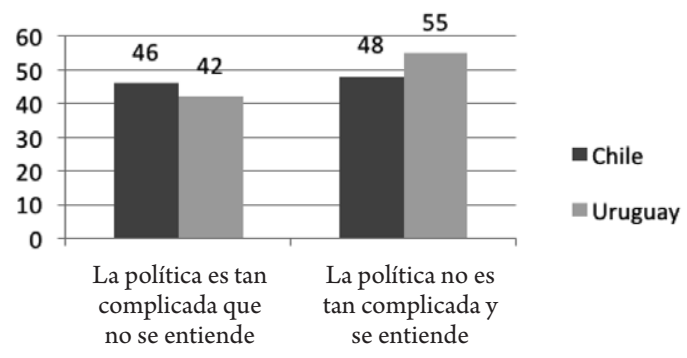

\section{Evidencia comparada respecto del papel de las campañas en los grados de eficacia política}

Entendemos, entonces, que existen variables y dimensiones que pueden llegar a ser trabajadas con acciones concretas para aumentar los niveles de eficacia personal en la política tanto interna como externa. En este sentido, nos hemos preguntado al inicio de este trabajo si las campañas electorales pueden tener algún tipo de incidencia en las dimensiones identificadas. De tener una respuesta positiva, sería necesario evaluar la conceptualización que históricamente se les ha dado a estas acciones políticas, las cuales reducen sus efectos usualmente a la dirección de preferencias o a la participación electoral.

Para tener una noción de los eventuales efectos que las campañas podrían tener desde el punto de vista de la eficacia política, resulta indispensable 
referirnos a los recientes estudios de Hansen y Pedersen (2014), quienes también entendieron que en el momento de estudiarlos debían ser mirados desde una perspectiva cívica, fundamental en un contexto democrático.

Independientemente de las hipótesis que pudieron trabajar los autores respecto de la incidencia de las campañas en diversos segmentos de población y sus correlaciones, lo que nos interesa, en esta oportunidad, hace relación simplemente con el aumento comprobado de los índices de las dimensiones medidas en los distintos grupos estudiados.

De esta forma, los autores concluyen que, en el contexto danés, las campañas electorales tuvieron consecuencias positivas respecto de la eficacia interna como externa, además de producir un aumento en los niveles de conocimiento, lo cual, en parte, condice con otras investigaciones anteriores (Finkel, 1985).

El estudio al que nos referimos se vuelve aún más sugerente y esencial para nuestro contexto si analizamos las dimensiones trabajadas por los autores (tabla 3), bastante similares a las ya utilizadas para evaluar la realidad chilena.

\section{Tabla 3. Preguntas respecto de índices de eficacia utilizados por Hansen y Pedersen (2014)}

\begin{tabular}{|c|l|}
\hline Dimensión & \multicolumn{1}{c|}{ Pregunta } \\
\hline \multirow{2}{*}{ Eficacia interna } & $\begin{array}{l}\text { A veces, la política es tan complicada que una persona como yo no puede realmente entender qué } \\
\text { está pasando. } \\
\text { En términos generales, no me parece tan difícil tomar una posición sobre cuestiones políticas. } \\
\text { Cuando los políticos debaten sobre economía, solo entiendo una pequeña parte de lo que dicen. } \\
\text { Gente como yo está calificada como para participar en discusiones políticas. } \\
\text { Gente como yo tiene opiniones políticas que valen la pena ser escuchadas. }\end{array}$ \\
\hline Eficacia externa & $\begin{array}{l}\text { A los políticos no les interesa realmente qué es lo que el votante piensa. } \\
\text { Usualmente puedes confiar en que los líderes políticos hacen lo mejor para el país. } \\
\text { Los políticos desperdician mucho dinero recaudado de impuestos. } \\
\text { Gente como yo no tiene influencia en lo que se decida en el Parlamento o en el Gobierno. }\end{array}$ \\
\hline
\end{tabular}

Claramente, los resultados no son extrapolables a la realidad nacional, pero la similitud de las dimensiones tratadas, los periodos de campaña, el contexto multipartidista y la metodología utilizada por los autores nos 
permitirían, al menos, elaborar de forma responsable una hipótesis basada en las conclusiones del estudio.

En este sentido, uno de los desafíos que se nos plantea dice relación con la posibilidad de medir con una metodología clara los eventuales cambios producidos en el contexto de campaña en los niveles de eficacia, ya que todo indicaría que los efectos debiesen ser similares a los expuestos por Hansen y Pedersen (2014).

\section{Consideraciones finales}

1) En Chile, así como en buena parte del mundo, nos encontramos inmersos en una crisis de confianza que ha dejado en evidencia los altos índices de desafección política de los últimos años. Esta realidad, que subyace incluso a la participación electoral, nos ha llevado a investigar alternativas que puedan significar una salida a la problemática. Es aquí precisamente donde se sitúan las actividades electorales como mecanismos que otorguen legitimidad y fortaleza al proceso democrático, lo cual — a nuestro juicio - requeriría una reestructuración del concepto mismo de 'campaña' en cuanto a sus efectos desde una perspectiva sistémica y cultural.

2) En ese mismo sentido, creemos que las campañas electorales, como acción política, han sido históricamente entendidas por la literatura en relación con la maximización de votos y, con ello, con la persuasión en la dirección de preferencias que lleven a ganar un proceso. Este entendimiento se evidencia en las distintas conceptualizaciones que se han propuesto a lo largo de la historia, las cuales dejan en un plano accesorio - o más bien invisible- a los diversos efectos beneficiosos que estas tienen en un contexto democrático.

Siguiendo esta línea, podríamos sostener responsablemente que la mayoría de los estudios realizados para evaluar la real necesidad de este tipo de acciones políticas se enmarcan en qué tanto persuaden, informan o comprometen al votante en virtud de obtener un escaño, pero claramente no existe el mismo grado de interés en conocer 
la incidencia de estos procesos en los índices de confianza, afección política y legitimación del proceso democrático.

3) Para conocer eventualmente los efectos que las campañas podrían tener en el proceso democrático en su sentido amplio, se hace necesario encontrar índices concretos que nos alejen de los conceptos válvula con que se suele tratar la apatía política. En la búsqueda de dimensiones concretas que nos permitan operacionalizar los índices de desafección y el papel que cumplen las campañas en ellos, surgen los conceptos de eficacia política planteados por Campbell, Gurin y Miller (1954). De esta forma, y a la luz de los distintos elementos que según los autores incidirían en la conformación del voto, nos centraríamos precisamente en las consecuencias que los actos de campaña podrían tener en los índices de eficacia personal en la política, entendida como un factor anterior y superior a la participación electoral. De esta forma, de incidir las campañas favorablemente en estos índices, podríamos inferir que la importancia de estos actos de campaña va más allá, incluso, de la mera participación en urnas, como legitimación de los procesos.

4) Por otro lado, si analizamos el contexto chileno a la luz de las dimensiones tratadas por Campbell, Gurin y Miller (1954), podríamos inferir que los índices de eficacia - tanto interna como externa - no son los mejores. En este sentido, independiente de la crisis de confianza que en estos minutos azota al país, los datos de distintos estudios demoscópicos sugieren que los índices han decaído en el tiempo, siendo peores que otros países con democracias más inclusivas del vecindario, como Uruguay.

De esta forma, entenderíamos que existen variables que afectan estos índices, las cuales eventualmente podrían ser trabajadas con distintas acciones.

5) ¿Serán las campañas electorales acciones políticas que podrían aumentar los niveles de eficacia? Para responder esta pregunta, nos referimos a los estudios de Hansen y Pedersen (2014), quienes, luego 
de una elaborada metodología de trabajo, sostienen que, al menos en Dinamarca, la campaña tuvo como consecuencia el aumento de los niveles de conocimiento y de los niveles de eficacia interna y externa. Para nosotros, esto es significativo por dos razones enunciadas por los autores. En primer lugar, por la carencia de investigaciones que aborden las campañas desde una perspectiva cívica (análisis que compartimos luego de estudiar la evolución del estudio), y en segundo lugar por los índices trabajados por los académicos, bastante similares a los que suelen utilizarse en Chile en diversos estudios demoscópicos, y con los cuales trabajamos en el contexto de la situación actual.

6) En esta misma línea, y sin querer extrapolar resultados a nuestra realidad nacional, creemos que es sensato comenzar a cambiar el paradigma como solemos entender los actos de campañas, para verlos como herramientas integrales que nos permitirían eventualmente tener una ciudadanía más cercana y comprometida con el sistema democrático.

Una nueva conceptualización —o cambio de paradigma en el estudio de las campañas - podría tener distintas consecuencias e implicancias. Por ejemplo, y tal como lo mencionan Hansen y Pedersen (2014), podría ser entendido de distinta manera el financiamiento estatal para estos actos electorales, entendiendo el papel crucial que tienen en la legitimación de los procesos y en la generación de una ciudadanía conocedora y consciente. En un segundo lugar, podría existir una nueva perspectiva en la búsqueda de herramientas que sean más eficaces a la hora de llegar a los distintos grupos objetivos, entendiendo también que los efectos esperables en cada uno varían, lo cual puede tener especial interés en el contexto de una esfera política digital (Jennings y Zeitner, 2003; Lupia y Baird, 2003; Kenski y Stroud, 2006; Lee, 2006; Effing, Van Hillegersberg, y Huibers, 2011).

Sabemos que es bastante improbable que el objeto de estudio cambie para quienes se dedican a ganar elecciones, pues lo más seguro es que para consultores el fin último siga siendo la maximización de votos. Aun así, creemos que la investigación y literatura no puede despreciar los efectos benéficos que estos actos podrían tener para el proceso 
democrático. De esta forma, sostenemos que basta con que se logre cercanía y empatía con el sistema para sentenciar que las campañas importan mucho más de lo que podríamos imaginar.

\section{Referencias}

Acock, A., Clarke, H. D. y Stewart, M. C. (1985). A new model for old measures: a covariance structure analysis of political efficacy. The Journal of Politics, 47(4), 1062-1084.

Ansolabehere, S. D., Iyengar, S. y Simon, A. (1999). Replicating experiments using aggregate and survey data: the case of negative advertising and turnout. American Political Science Review, 93(4), 901-909.

Arceneaux, K. (2006). Do campaigns help voters learn? A cross-national analysis. British Journal of Political Science, 36(1), 159-173.

Ashworth, S. y Clinton, J. D. (2007). Does advertising exposure affect turnout? Quarterly Journal of Political Science, 2(1): 27-41.

Atkin, C. y Heald, G. (1976). Effects of political advertising. The Public Opinion Quarterly, 40(2), 216-228.

Balch, G. I. (1974). Multiple indicators in survey research: the concept "sense of political efficacy”. Political Methodology, 1(2), 1-43.

Banducci, S. A. y Karp, J. A. (2003). How elections change the way citizens view the political system: campaigns, media effects and electoral outcomes in comparative perspective. British Journal of Political Science, 33(3), 443-467.

Brians, C. L. y Wattenberg, M. P. (1996). Campaign issue knowledge and salience: Comparing reception from TV commercials, TV news and newspapers. American Journal of Political Science, 40(1), 172-193. 
Campbell, A., Gurin, G. y Miller, W. E. (1954). The voter decides. Oxford, Inglaterra: Row, Peterson, and Co.

Canel, M. J. (1998). Los efectos de las campañas electorales. Comunicación y Sociedad, 11(1), 47-67.

Caprara, G. V., Vecchione, M., Capanna, C. y Mebane, M. (2009). Perceived political self-efficacy: Theory, assessment, and applications. European Journal of Social Psychology, 39(6), 1002-1020.

Claassen, R.L. (2011). Political awareness and electoral campaigns: Maximum effects for minimum citizens? Political Behavior, 33(2), 203-223.

Coleman, J. J. y Manna, P. F. (2000). Congressional campaign spending and the quality of democracy. Journal of Politics, 62(3), 757-789.

Converse, P. E. (1972). Change in the American electorate. En A. Campbell y P. E. Converse (eds.), The human meaning of social change (pp. 263-337). Nueva York: Russell Sage Foundation.

Dalager,J. K. (1996). Voters, issues, and elections: Are the candidates' messages getting through? The Journal of Politics, 58(2), 486-515.

Domínguez, J. (1997). Latin America’s crisis of representation. Foreign Affairs, 76, 100-113.

Effing, R., Van Hillegersberg, J. y Huibers, T. (2011). Social media and political participation: Are Facebook, Twitter and YouTube democratizing our political systems? Electronic Participation, 6847, Lecture Notes in Computer Science, 25-35.

Finkel, S. E. (1985). Reciprocal effects of participation and political efficacy: A panel analysis. American Journal of Political Science, 29(4), 891-913.

Fiorina, Morris P. (1981). Retrospective voting in American national elections. New Haven, CT: Yale University Press. 
Freedman, P., Franz, M. y Goldstein, K. (2004). Campaign advertising and democratic citizenship. American Journal of Political Science, 48(4), 723-741.

García Beaudoux, V. (2004). Campañas electorales y sus efectos sobre el voto: análisis de la campaña electoral presidencial 2003 en Argentina. Psicología Política, 28, 7-26.

Goldstein, K.y Ridout, T. N. (2004). Measuring the effects of televised political advertising in the United States. Annual Review of Political Science, 7, 205-226.

Graber, D. A. (1996). The "new" media and politics: What does the future hold? Political Science \& Politics, 29(1), 33-36.

Hansen, K. M. y Pedersen, R. T (2014). Campaigns matter: How voters become knowledgeable and efficacious during election campaigns. Political Communication, 31(2), 303-324.

Huber, G. A. y Arceneaux, K. (2007). Identifying the persuasive effects of presidential advertising. American Journal of Political Science, 51(4), 957-977.

Jennings, M. K. y Zeitner, V. (2003). Internet use and civic engagement: A longitudinal analysis. Public Opinion Quarterly, 67(3), 311-334.

Jung, N., Kim, Y. y De Zúñiga, H. G. (2011). The mediating role of knowledge and efficacy in the effects of communication on political participation. Mass Communication and Society, 14(4), 407-430.

Kahn, K. F. y Kenney, P. J. (2000). How negative campaigning enhances knowledge of Senate elections. En James A. Thurber, Candice J. Nelson y David A. Dulio (eds.), Crowded airwaves: campaign advertising in elections (pp. 65-95). Washington: Brookings Institution Press. 
Kenski, K. y Stroud, N. J. (2006). Connections between Internet use and political efficacy, knowledge, and participation. Journal of broadcasting \& Electronic Media, 50(2), 173-192.

Key, V. O. (1966). The responsible electorate: Rationality in presidential voting 1936-1960. Cambridge, MA: Harvard University Press.

Klapper, J. T. (1957). What we know about the effects of mass communication: the brink of hope. Public Opinion Quarterly, 21(4), 453-474.

Klapper, J. T. (1960). The effects of mass communication. An analysis of research on the effectiveness and limitations of mass media in influencing the opinions, values, and behavior of their audiences. Nueva York: The Free Press.

Krasno, J. S. y Green, D. P. (2008). Do televised presidential ads increase voter turnout? Evidence from a natural experiment. The Journal of Politics, 70(1), 245-261.

Lane, R. E. (1959). Political life: How and why do people get involved in politics? New York, NY: Free Press.

Lau, R. R. y Redlawsk, D. P. (1997). Voting correctly. American Political Science Review, 91(3), 585-598.

Lazarsfeld, P. F., Berelson, B. y Gaudet, H. (1948). The peopleos choice: How the voter makes up his mind in a presidential campaign. Nueva York: Columbia University Press.

Lee, K. M. (2006). Effects of Internet use on college students' political efficacy. Cyberpsychology \& Behavior, 9(4), 415-422.

Lippmann, W. (2003 [1922]).La opinión pública. Madrid: Cuaderno de Lange.

Lupia, A. y Baird, Z. (2003). Can web sites change citizens? Implications of web white and blue 2000. Political Science and Politics, 36(1), 77-82. 
Maarek, P. J. (2011). Campaign communication and political marketing. John Wiley \& Sons.

Martínez i Coma, F. (2005). ¿Cuáles fueron los efectos de la campaña electoral española del 2000? Revista Española de Investigaciones Sociológicas, 112(1), 231-257.

McGinnis, J. (1968). The selling of the president. Nueva York: Trident.

Moreno Martínez, C. (2010). El efecto de la campaña para las elecciones generales españolas de 2008 sobre la información política y la participación electoral de los votantes: ¿se puede hablar de una función de legitimación de las campañas electorales? Revista Española de Ciencia Política, 24, 53-81.

Morrell, M. E. (2003). Survey and experimental evidence for a reliable and valid measure of internal political efficacy. Public Opinion Quarterly, 67(4), 589-602.

Niemi, R. G., Craig, S. C. y Mattei, F. (1991). Measuring internal political efficacy in the 1988 National Election Study. American Political Science Review, 85(4), 1407-1413.

Norris, P. (2001). ¿Un círculo virtuoso? El impacto de las comunicaciones políticas en las democracias post-industriales. Revista Española de Ciencia Política, 4(1), 7-33.

Norris, P. (2002). Do campaign communications matter for civic engagement? American elections from Eisenhower to George Bush. En D. M. Farrell y R. Schmitt-Beck (eds.), Do political campaigns matter? Campaign effects in elections and referendums (pp. 119-136). Nueva York: Routledge/ECPR Studies in European Political Science.

Norris, P., Frank, R. W. y i Coma, F. M. (2013). Assessing the quality of elections. Journal of Democracy, 24(4), 124-135. 
Pinkleton, B. E., Austin, E. W. y Fortman, K. K. (1998). Relationships of media use and political disaffection to political efficacy and voting behavior. Journal of Broadcasting \& Electronic Media, 42(1), 34-49.

Popkin, S. I. (1991). The reasoning voter: Communication and persuasion in presidential campaigns. Chicago: University of Chicago Press.

Rodríguez-Polo, X. R. (2011). Los efectos de la comunicación de masas de Joseph T. Klapper. Razón y Palabra, 75.

Sides, J.y Vavreck, L. (2014). The gamble: Choice and chance in the 2012 presidential election. Princeton University Press.

Torcal, M. (2006). Political disaffection and democratization history in new democracies. En Political disaffection in contemporary democracies: social capital, institutions and politics (pp. 157-189). Londres: Routledge.

Tocal, M. y Montero, J. R. (2006). Political disaffection in contemporary democracies. Social capital, institutions, and politics. Londres/Nueva York: Routledge.

Wattenberg, M. P. y Brians, C. L. (1999). Negative campaign advertising: Demobilizer or mobilizer? American Political Science Review, 93(4), 891-899.

Zhang, W., Johnson, T. J., Seltzer, T. y Bichard, S. L. (2009). The revolution will be networked: The influence of social networking sites on political attitudes and behavior. Social Science Computer Review.

Zhao, X. y Chaffee, S. H. (1995). Campaign advertisements versus television news as sources of political issue information. Public Opinion Quarterly, 59(1), 41-65. 